Ann. Biol. anim. Bioch. Biophys., 1979, 19 (3 B), 781-785.

\title{
Effet des glucides ingérés sur l'absorption et la rétention de minéraux toxiques
}

\author{
par D. BOUVET, J. PESTIS, Alice FOURNIER
}

Laboratoire de Physiologie, U.E.R. Sciences Phormaceutiques ef Métabolisme minéral des Mammifères, E.P.H.E., ERA-CNRS 070572, 92290 Chôfenay-Malabry.

Summary. Effect of ingested carbohydrates on the absorption and retention of toxic minerals.

Lactose and many carbohydrates increase the absorption of various biolcgically interesting minerals ( $\mathrm{Ca}, \mathrm{Fe}, \mathrm{Cu}, \mathrm{Zn}, \mathrm{Mg}, \mathrm{Mn}, \mathrm{Co}$ ) by 2 to 4 -fold. These minerals are extensively stored during the long intake of a glucidic diet, and their possible harmful efiects are unknown. The influence of carbohydrates upon the absorption and retention of toxic minerals has been little studied. Natural milk intake by a very receptive young animal increased the retention of $\mathrm{Pb} 33$ to 57 -fold and that of $\mathrm{Cd}$ by up to 17 times; $\mathrm{Hg}$ absorption also increased. A high sucrose of lactose diet increased $V$ toxicity. In humans, $C d$ concentration increased during the first 3 years more than 200 times, that is one-third of the total Cd accumulated during a lifetime. The toxic result of $\mathrm{Cd}$ is beginning to be known since the work of Parizek and Záhor. Our study concerns the effect of sorbitol upon the retention and absorption of cadmium. Four-month old rats, fasted for 15 hours, were given through an oesophagus probe, $2 \mathrm{ml}$ of a watery solution containing $2 \mathrm{ppm} \mathrm{Cd}$ (sulfate) and $1.5 \mu \mathrm{Ci}{ }^{109} \mathrm{Cd}$, a solution which would be $0,50,100,150,200$ or $300 \mathrm{mM}$ of sorbitol ; 24 hours after intake, Cd absorption was determined according to the radioactivity of the ${ }^{109} \mathrm{Cd}$ retained in the liver and kidneys. Hepatic radioactivity alone steadily increased. Without sorbitol, this radioactivity was 10.9 p. 1000 of the administered dose. It rose to 17.2 p. 1000 for the $200 \mathrm{mM}$ sorbitol solution. The graph representing the fixation of $\mathrm{Cd}$ in relation to sorbitol concentration, was similar to those obtained after simultaneous intake of a carbohydrate and a mineral ( $\mathrm{Ca}$ or $\mathrm{Fe}$ ).

\section{Introduction.}

L'essentiel des études de l'influence des glucides sur l'absorption minérale a porté tout principalement sur le transport couplé des ions sodium et du D-glucose. Le rôle des glucides sur l'absorption des alcalino-terreux et d'autres minéraux a été bien moins étudié.

A la différence du sodium, ce sont surtout la grande majorité des glucides non transportés activement et relativement peu absorbables qui facilitent l'absorption minérale en général. Mais quelques glucides activement absorbés accroissent également cette absorption (Bouvet, 1977 ; Fournier et al., 1973).

Le lactose et de nombreux glucides augmentent l'absorption de divers minéraux d'intérêt biologique : $\mathrm{Ca}, \mathrm{Fe}, \mathrm{Cu}, \mathrm{Zn}, \mathrm{Mg}, \mathrm{Mn}, \mathrm{Co}$ (Bouvet, 1977 ; Fournier et al., 1974 ; 
Fournier et al., 1973). Une longue ingestion de régime riche en certains glucides conduit, cependant, à une forte accumulation de la plupart des métaux précités dont le possible effet nocif reste à préciser.

L'influence des glucides sur l'absorption et la rétention de minéraux toxiques a été abordée et mériterait d'être poursuivie, d'autant que, dans le passé, des erreurs ont été commises. En effet, des aliments riches en glucides furent utilisés pour empêcher une contamination (possible) en minéraux toxiques. Par exemple, à la suite de nombreuses observations, on pensait que le lait pouvait prévenir l'intoxication au $\mathrm{Pb}$; ef même, pendant longtemps, la consommation de lait était recommandée au personnel travaillant dans l'industrie du $\mathrm{Pb}$. En fait, ce traitement favorisait la fixation du $\mathrm{Pb}$ dans l'organisme parce qu'il augmentait son absorption au niveau de l'intestin (Kello ef Kostial, 1973). Ce supplément alimentaire améliorait cependant l'étał général des ouvriers mal nourris et il en découlait une meilleure résistance au saturnisme (Stephens ef Waldron, 1975).

Le lait accroît d'autant plus l'absorption ef la rétention de Cd que l'animal est jeune (Kello et Kostial, 1977). L'hypothèse serait qu'un des constituants du lait, qui pourrait être le lactose, influencerait l'absorption du Pb (Kello et Kostial, 1973) et celle du Cd en chélatant ce métal.

Si un régime glucidique augmente de 2 à $\mathbf{4}$ fois l'absorption de nombreux minéraux d'intérêt biologique, ce même régime multiplie de 10 à 60 fois la rétention de minéraux toxiques, l'accroissement de la rétention étant dû à l'augmentation de l'absorption intestinale de ces minéraux sous l'effet de ce régime (Kello et Kostial, 1973, 1977). Ainsi, chez le jeune animal, le loit augmente de 33 à 57 fois la rétention du Pb (Kello et Kostial, 1973) et jusqu'à 17 fois celle du Cd (Kello et Kostial, 1977) ; l'absorption du $\mathrm{Hg}$ est aussi fortement accrue. Un régime riche en lactose augmente la toxicité du V (Hafez et Kratzer, 1976).

Le jeune Mammifère est très réceptif à l'intoxication minérale à cause de son âge et de la nature du régime puisqu'il ingère tout naturellement du lait. Chez l'homme, la concentration de $\mathrm{Cd}$ augmente durant les trois premières années de plus de 200 fois, soit le tiers du $C d$ total accumulé durant une vie (Henke et al., 1970). Certains ustensiles de cuisine libèrent des quantités importantes de $\mathrm{Cd}$, les boîtes de lait condensé contiennent en solution du Cd, du $\mathrm{Pb}$ et du $\mathrm{Sn}$ (Karhausen, 1974).

Notre étude porte sur l'effet du sorbitol sur la rétention et l'absorption du Cd. Le sorbitol, comme le lactose, se trouve en grandes quantités dans la nature, et est utilisé dans diverses préparations pharmaceutiques. Ainsi, des petites doses de sous-nitrate de $\mathrm{Bi}$ ont souvent un effet constipant que l'on peut corriger par addition de sorbitol.

\section{Conditions expérimentales.}

Des rats de 4 mois, à jeûn durant $15 \mathrm{~h}$, reçoivent par sonde osophagienne $2 \mathrm{ml}$ d'une solution aqueuse à $2 \mathrm{ppm}$ de $\mathrm{Cd}$ (sulfate), $1,5 \mu \mathrm{Ci}$ de ${ }^{109} \mathrm{Cd}$, solution pouvant être $0,50,100,150,200$ ou $300 \mathrm{mM}$ en sorbitol. Vingt-quatre heures après l'ingestion, la radioactivité de ${ }^{109} \mathrm{Cd}$ retenue par la foie et les reins est mesurée, le rein et surtout le foie étant les organes qui fixent la majeure partie du Cd absorbé par l'organisme (Cousins ef al., 1973 ; Fox et al., 1971). 


\section{Résultats.}

Il apparaît clairement sur le tableau 1 que la fixation de ${ }^{109} \mathrm{Cd}$ par le foie est augmentée lorsque la quantité de sorbitol ingérée est accrue. Sans sorbitol, cette radioactivité est de 10,9 p. 1000 de la dose administrée. Avec le sorbitol, elle s'élève jusqu'à 17,2 p. 1000 pour la solution à $200 \mathrm{mM}$; mais à $300 \mathrm{mM}$ en sorbitol, la rétention en ${ }^{109} \mathrm{Cd}$ ne semble plus augmentée par rapport à celle obtenue avec $200 \mathrm{mM}$.

TABLEAU 1

Influence du sorbifol sur la fixation de ${ }^{109} \mathrm{Cd}$ par le foie

\begin{tabular}{ccccc}
\hline $\begin{array}{c}\text { Concentration } \\
\text { en sorbitol } \\
\text { de la } \begin{array}{c}\text { Teneur } \\
\text { (mM) }\end{array}\end{array}$ & $\begin{array}{c}\text { Ecart type } \\
\text { moyenne } \\
\text { en }{ }^{109} \mathrm{Cd}\end{array}$ & $\mathrm{N}$ & $\begin{array}{c}\text { Ecart typé } \\
\mathrm{s} / \sqrt{\mathrm{N}}\end{array}$ & $\begin{array}{c}\text { Degré de } \\
\text { signification } \\
\text { (Test de Fischer) }\end{array}$ \\
\hline 0 & $10,96 *$ & $13 * *$ & 0,65 & $\mathrm{NS}$ \\
50 & 13,77 & $5 i$ & 0,92 & $\mathrm{NS}$ \\
100 & 14,17 & 6 & 1,82 & $\mathrm{P} \leqslant 0,05$ \\
150 & 15,37 & 6 & 1,43 & $\mathrm{P} \leqslant 0,01$ \\
200 & 17,20 & 6 & 2,23 & $\mathrm{P} \leqslant 0,05$ \\
300 & 14,47 & 6 & 1,99 & \\
\hline
\end{tabular}

* Teneur moyenne en ${ }^{109} \mathrm{Cd}$ par foie entier (p. 1000 de la dose de ${ }^{109} \mathrm{Cd}$ ingéré).

** Nombre de rats par lot.

Par contre le sorbitol n'accroît pas la fixation de $C d$ par le rein puisque la rétention globale des deux reins est de 1,8 p. 1000 de la dose de ${ }^{109} \mathrm{Cd}$ administrée sans sorbitol. Pour 100, $200 \mathrm{mM}$ en sorbitol, cette même rétention est d'environ 2 p. 1000.

La courbe de la figure 1 montre l'augmentation de la fixation de ${ }^{109} \mathrm{Cd}$ par le foie sous l'action du sorbitol. Pour les faibles concentrations en sorbitol (50 à $100 \mathrm{mM}$ ) la rétention hépatique est augmentée mais de façon non significative. Des concentrations de 150, 200, $300 \mathrm{mM}$ en sorbitol augmentent significativement la fixation de ${ }^{109} \mathrm{Cd}$ par le foie. Une quantité de sorbitol supérieure à $200 \mathrm{mM}$ ne permet plus d'accroître cette radioactivité au-dessus de 56,9 p. 100. S'agit-il d'une limite, d'autres expériences répondront peut-être à ce problème.

FIG. 1. - Augmentation de la fixation de ${ }^{100} \mathrm{Cd}$ par le foie sous l'effet du sorbitol. Chaque point représente la moyenne d'un lot $\pm \mathrm{Sm}$.

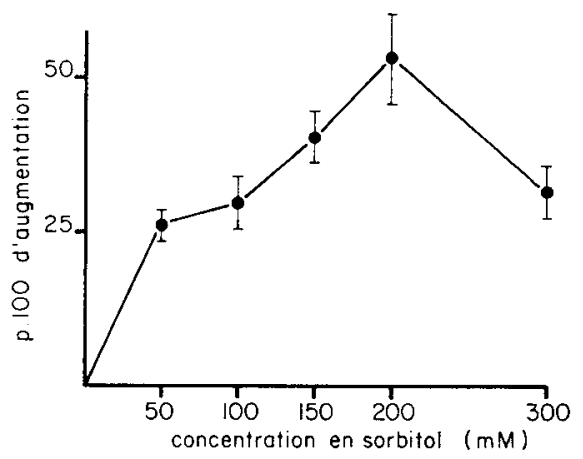




\section{Discussion et conclusion.}

Ainsi la rétention du Cd par le foie augmente avec la concentration de la solution en sorbitol. La rétention hépatique et rénale ont été proposées comme moyen d'apprécier les variations de l'absorption intestinale du Cd (Hamilton et Smith, 1978).

De semblables phénomènes ont pu être observés en ce qui concerne l'effet de la concentration du composé glucidique sur la grandeur de l'absorption intestinale du Fe in vivo et sur la grandeur de la fixation de ce métal sur l'hématie in vifro (Bouvet, 1977). II en est de même pour l'absorption du Ca in vivo (Dupuis et al., 1976) et celle du Cu in vitro (Ohara ef al., 1969), ce qui suggère que le glucide, par un même mécanisme, influence l'absorption minérale.

En ce qui concerne le Fe, l'analyse des courbes de fixation sur l'hématie du Fe mis en présence du glucide évoque la notion d'un site actif de transfert propre au métal cinétique de type enzymatique (Bouvet, 1977). De même, le fait que le glucide augmente l'absorption du $\mathrm{Ca}$ et que le phosphate la diminue selon des courbes à allure michaélienne, conduit également à la notion de transfert à caractère enzymatique (Fournier ef Dupuis, 1975). La phosphatase alcaline intestinale pourrait être en cause, le glucide intervenant en qualité d'accepteur de phosphate (Dupuis et al., 1977).

Un tel schéma reste-t-il valable en ce qui concerne l'absorption des métaux toxiques?

Commission CNERNA Digestion-Absorption/Association des Physiologistes,

Paris 5-6 octobre 1978.

\section{Références}

BOUVET D., 1977. Efudes du mécanisme d'action des glucides sur l'absorption ef l'utilisation du fer. Thèse Dr ès-Sci. nat., Paris.

COUSINS R. J., BARBER A. K., TROUT J. R., 1973. Cadmium toxicity in growing swine. J. Nutr., 103, 964-972.

DUPUIS Y., FOURNIER A., FOURNIER P., 1976. Etude, en anses intestinales, in situ, de la modulation par un glucide et par un phosphate de l'absorption du calcium. C.R. Soc. Biol., 170, $304-309$.

DUPUIS Y., DIGAUD A., FONTAINE N., 1977. Etude des effets du sorbifol sur l'activité des phosphatases alcalines isolées de diverses régions de l'intestin grêle du rat. C.R. Soc. Biol., 171, 294-302.

FOURNIER A., DIGAUD A., FOURNIER P., 1974. Augmentation, chez le rat, de l'absorption et de la rétention du cobalt ${ }^{58}$ sous l'effet d'une ingestion simultanée de lactose. C.R. Soc. Biol., 168, 244-247.

FOURNIER P., DUPUIS Y., FONTAINE N., SEVETTE A. M., 1973. Spécificité moléculaire ef activité des glucides sur l'absorption du calcium. J. Physiol., Paris, 66, 473-477.

FOURNIER P., DUPUIS Y., 1975. La modulation de l'absorption intestinale du calcium. J. Physiol., Paris, 70, 479-491.

FOX M. R. S., FRY B. E., HARLAND B. F., SCHERTEL M. E., WEEKS C. E., 1971. Effect of ascorbic acid on cadmium toxicity in the young coturnix. J. Nutr., 101, 1295-1306.

HAFEZ Y. S. M., KRATZER F. H., 1976. The effect of diet on the toxicity of vanadium. Poultry Sci., 55, 918-922.

HAMILTON D. L., SMITH M. W., 1978. Inhibition of intestinal calcium uptake by cadmium and the effect of a low calcium diet on cadmium retention. Environ. Res., 15, 175-184.

HENKE G., SACHS H. W., BOHM G., 1970. Cadmium-Bestimmungen in Leber und Nieren von Kindern und Jugendlichen durch Neutronenaktivierungs analyse. Arch. Toxikol., 26, 8-16. 
KARHAUSEN L. R., 1974. L'absorption intestinale du cadmium et du mercure. In : Problems of the contamination of man and his environment by mercury and cadmium. Eur. Coll. Comm. Eur. Commun. Luxembourg.

KELLO D., KOSTIAL K., 1973. The effect of milk diet on lead metabolism in rats. Environ. Res., 6 , 355-360.

KELLO D., KOSTIAL K., 1977. Influence of age and milk diet on cadmium absorption from the gut. Toxicol. appl. Pharmocol., USA, 40, 277-282.

OHARA M., PEIRIS B., IKUZAWA M., UESAKA S., 1969. Effect of sugars on the absorption of copper from the small intestine of rat. Jap. J. Zootech. Sci., 40, 12-17.

PARIZEK Z., ZAHOR Z., 1956. Effect of cadmium salts on testicular tissue. Nature, London, 177, 1036.

STEPHENS R., WALDRON H. A., 1975. The influence of milk diet and related dietary constituents on lead metabolism. Fed. Cosmef. Toxicol., 13, 555-563. 